\title{
Outcomes of contemporary emergency open surgery for type A acute aortic dissection in elderly patients
}

\author{
Akihito Matsushita, MD, ${ }^{\mathrm{a}}$ Minoru Tabata, MD, MPH, ${ }^{\mathrm{a}}$ Toshihiro Fukui, MD, ${ }^{\mathrm{a}}$ Yasunori Sato, PhD, ${ }^{\mathrm{b}}$ \\ Shigefumi Matsuyama, MD, ${ }^{\mathrm{c}}$ Tomoki Shimokawa, $\mathrm{MD},{ }^{\mathrm{c}}$ and Shuichiro Takanashi, MD ${ }^{\mathrm{a}}$
}

Objectives: We sought to evaluate surgical outcomes of type A acute aortic dissection in elderly patients.

\begin{abstract}
Methods: Between January 2004 and July 2011, 422 patients underwent emergency open surgery for type A acute aortic dissection at our institution. Of those, 124 patients who were $\geq 75$ years (mean age, $78.6 \pm 3.4$ years) were reviewed. We also reviewed 26 patients ( $\geq 75$ years old) who were diagnosed with acute aortic dissection at our institution during the same period but who did not undergo surgery. We analyzed early and late outcomes of surgical and nonsurgical patients.
\end{abstract}

\begin{abstract}
Results: The operative mortality was $4.8 \%(6 / 124)$, and the incidences of stroke and prolonged hospital stay (>30 days) were $17.7 \%(22 / 124)$ and $20.1 \%$ (25/124), respectively. The actuarial survivals at 1,3 , and 5 years were $89.3 \%, 84.7 \%$, and $79.1 \%$, respectively. Predictors of stroke are preoperative cardiopulmonary resuscitation (odds ratio, $17.5 ; 95 \%$ confidence interval, 3.1-98.9; $P=.001$ ) and previous cardiac surgery (odds ratio, $14.0 ; 95 \%$ confidence interval, $1.2-164.7 ; P=.036$ ). The 30 -day or in-hospital mortality of patients who were indicated for surgery but refused surgery was $63.6 \%(7 / 11)$.
\end{abstract}

Conclusions: Emergency open surgery for type A acute aortic dissection in elderly patients resulted in a low mortality but high incidences of stroke and prolonged hospital stay. Preoperative cardiopulmonary resuscitation and previous cardiac surgery were significant predictors of stroke. Emergency surgery is still the primary option for most elderly patients with acute aortic dissection. (J Thorac Cardiovasc Surg 2014;147:290-4)

Type A acute aortic dissection (AAD) is a life-threatening emergency that carries a high mortality rate without surgical treatment. ${ }^{1}$ Immediate surgery for repair of AAD has been recommended to halt extension, improve branch flow, and prevent aortic rupture or cardiac tamponade. Surgical outcomes of AAD have been improving with increasing experience and advances in diagnosis, surgical techniques, and perioperative management. ${ }^{2}$ However, most articles have reported consistently that advanced age still remains a significant determinant of mortality after surgery for AAD..$^{3-7}$ Also, advanced age has been shown to be associated with neurologic complications after surgical treatment of $\mathrm{AAD}^{3,7}$ On the other hand, analyses of the International Registry of Acute Aortic Dissection and the German Registry for Acute Aortic Dissection Type A have shown that conservative treatment is more likely to result in death than surgical treatment for AAD even in elderly

From the Department of Cardiovascular Surgery, ${ }^{\text {a }}$ Sakakibara Heart institute, Tokyo, Japan; the Clinical Research Center, ${ }^{\text {b }}$ Graduate School of Medicine, Chiba University, Chiba, Japan; and the Department of Cardiovascular Surgery, ${ }^{\mathrm{c}}$ Teikyo University Hospital, Tokyo, Japan.

Disclosures: Authors have nothing to disclose with regard to commercial support. Received for publication Sept 7, 2012; revisions received Oct 3, 2012; accepted for publication Nov 6, 2012; available ahead of print Dec 10, 2012.

Address for reprints: Minoru Tabata, MD, Department of Cardiovascular Surgery, Sakakibara Heart Institute, 3-16-1 Asahi-cho, Fuchu-shi, Tokyo 183-0003, Japan (E-mail: mtabata@post.harvard.edu).

0022-5223/\$36.00

Copyright $(2) 2014$ by The American Association for Thoracic Surgery http://dx.doi.org/10.1016/j.jtcvs.2012.11.007 patients. ${ }^{6,7}$ These findings endorse that surgical therapy for AAD should not be denied on the basis of advanced age alone. In the absence of practice guidelines, the optimal management of elderly patients with AAD still remains poorly defined.

Thorough operative risk assessment is difficult in emergency situations. Risk scoring systems are useful to predict surgical mortality and morbidity; however, it is often difficult to collect all information necessary for scoring in emergency situations. In addition, other factors such as frailty and family/social support need to be considered, as well as comorbidities in cases of elderly patients.

In this study, we evaluated early and late outcomes of surgical and nonsurgical elderly patients with AAD and explored predictors of stroke after emergency open surgery in this group of patients.

\section{METHODS}

\section{Patient Population}

This study was approved by the institutional review board, and a waiver of informed consent was obtained. Between January 2004 and July 2011, 422 patients underwent emergency open surgery for type A AAD at Sakakibara Heart Institute. Of those, we reviewed 124 patients who were $\geq 75$ years old. We also reviewed 26 patients ( $\geq 75$ years old) who were diagnosed with type A AAD at our institution during the same period but who did not undergo surgery.

The diagnosis was made with contrast computed tomography in all patients. Type A AAD was defined by visualizing an intimal flap separating 2 lumens in the ascending aorta. The decision-making process for our cohort is shown in Figure 1. Patients with unsuccessful cardiopulmonary 


\section{Abbreviations and Acronyms \\ $\mathrm{AAD}=$ acute aortic dissection \\ $\mathrm{CPR}=$ cardiopulmonary resuscitation \\ $\mathrm{TAR}=$ total arch replacement}

resuscitation (CPR) and comatose patients without pupillary light reflex were considered inoperable. According to our routine practice, we chose conservative treatment for patients with a fully thrombosed false lumen (noncommunicating aortic dissection or intramural hematoma) and an ascending aorta with a diameter $<45 \mathrm{~mm}$ regardless of age. ${ }^{8}$ Otherwise, we offered a surgical option to patients or their family members, explaining individual risks and benefits of the surgery to them.

In surgical patients, the mean age was $78.6 \pm 3.4$ years, the oldest patient was 91 years old, and 94 patients $(76 \%)$ were women. Thirty-one patients $(25 \%)$ had cardiac tamponade, 8 patients $(6.5 \%)$ had preoperative $\mathrm{CPR}$, and 3 patients $(2.4 \%)$ had previous cardiac surgery. Preoperative characteristics of surgical patients are shown in Table 1.

In nonsurgical patients, the mean age was $81.8 \pm 5.5$ years old and 17 $(65 \%)$ were women. Four patients did not undergo surgery because of unsuccessful CPR or comatose condition without pupillary light reflex, 11 patients did not undergo surgery because of a fully thrombosed false lumen and an ascending aorta with a diameter $<45 \mathrm{~mm}$, and 11 patients refused surgery. Those 11 patients who refused surgery were significantly older (mean age, $83.9 \pm 6.1$ years) than surgical patients. Also, most of them were very frail according to their medical records, although we did not evaluate frailty in an objective fashion. Baseline characteristics of patients who refused surgery are compared with those of surgical patients in Table 1.

\section{Data Collection}

Perioperative data were collected from patients' medical records. Cardiac tamponade was defined as a cardiogenic shock with a systolic blood pressure of $\leq 90 \mathrm{~mm} \mathrm{Hg}$, associated with pericardial effusion confirmed by computed tomography and/or echocardiography. Stroke was defined as a central neurologic deficit persisting for $>72$ hours after surgery. All strokes were confirmed by computed tomography or magnetic resonance imaging. Respiratory failure was defined as a requirement for mechanical ventilation for $>48$ hours postoperatively. Operative mortality was defined as any death within 30 days after surgery or before discharge. The median follow-up period was 27 months (range, 0-95 months).

\section{Operative Strategy and Procedure}

All operations were performed through a median sternotomy; the initial arterial cannula was placed mostly in the femoral artery. The left ventricular apex or axillary artery was cannulated if the femoral artery was unavailable. Our strategy of selection of the cannulation site has been described previously. ${ }^{9}$ In all surgical patients, a combination of antegrade and retrograde cardioplegia was used, and patients were cooled to $25^{\circ} \mathrm{C}$ for circulatory arrest. A single-branch prosthesis was used in ascending aortic or hemiarch replacement; a 4-branch prosthesis was used in total arch replacement (TAR).

The proximal aorta was repaired with gelatin resorcinol formaldehyde glue and double strips (vascular graft strip inside and Teflon felt strip outside) at the level of the sinotubular junction. When there was an intimal tear in the aortic root or the aortic root was dilated, the aortic root was replaced without using glue.

In ascending aortic or hemiarch replacement, the distal aortic repair and anastomosis was performed under hypothermic circulatory arrest with retrograde cerebral perfusion from the superior vena cava. The distal aorta was repaired with double Teflon felt strips and then anastomosed to the vascular graft. After the distal anastomosis, whole-body circulation was resumed through the branch of the prosthesis and the patient was fully rewarmed to $35^{\circ} \mathrm{C}$. Proximal anastomosis was performed during rewarming.

Between January 2004 and August 2006, we used the arch-first technique with retrograde cerebral perfusion in TAR $(n=7)$. Subsequently, in 2006, we introduced the stepwise distal anastomosis technique with antegrade selective cerebral perfusion and separate lower body perfusion $(\mathrm{n}=11) .{ }^{10}$ The details of our current TAR techniques have been reported previously. ${ }^{10}$

\section{Statistical Analysis}

Summary statistics were constructed using frequencies and proportions for categorical data, and mean \pm standard deviation or median, if appropriate, for continuous variables. Univariate analyses were carried out using a $t$ test or Mann-Whitney $U$ test for continuous variables and a Fisher exact test for categorical variables.

For time-to-event outcomes, the times elapsed until a first event were compared using the log-rank test, whereas the Kaplan-Meier method was used to estimate the absolute risk of each event for each group. We explored predictors of stroke using a stepwise multivariate logistic regression model. Predictors included in the initial model were age, female sex, cardiac tamponade, preoperative CPR, preoperative neurologic deficit, myocardial ischemia, limb ischemia, previous cardiac surgery, preoperative hematocrit and serum creatinine levels, and TAR. All data were analyzed according to the intent-to-treat principle, and all comparisons were planned and the tests were 2 sided. A $P$ value $<.05$ was considered to indicate a statistically significant difference. All statistical analyses were performed by using the PASW 18.0 (IBM-SPSS Inc, Chicago, Ill).

\section{RESULTS \\ Operative Outcomes}

We performed ascending aortic replacement or hemiarch replacement in 106 patients $(85.5 \%)$, TAR in 18 patients $(14.5 \%)$, and concomitant procedures in 28 patients $(22.6 \%)$ : Bentall operation in 9 patients, coronary artery bypass grafting in 11 patients, aortic valve replacement in 4 patients, and other procedures in 6 patients.

Operative mortality was $4.8 \%(6 / 124)$. Twenty-two patients $(17.7 \%)$ had newly developed stroke and 27 patients $(21.8 \%)$ had respiratory failure. Median intensive care unit stay was 4 days and median length of hospital stay was 21 days; 25 patients $(20.1 \%)$ stayed in the hospital for $>30$ days after surgery. The operative mortality of 39 patients with fully thrombosed false lumen was $0 \%$. Operative data and outcomes are shown in Table 2.

The Kaplan-Meier analysis estimated that actuarial 1-, 3-, and 5-year survivals were $89.3 \%, 83.6 \%$, and $79.1 \%$ (Figure 2). During the follow-up period, 10/22 patients who had stroke presented with improved neurologic function and eventually recovered activities of daily living to some extent. Six patients became bedridden.

\section{Predictors of Stroke}

A stepwise multivariate logistic regression model showed that significant predictors of stroke were preoperative CPR (odds ratio, 17.5; 95\% confidence interval, 3.198.9; $P=.001$ ) and previous cardiac surgery (odds ratio, $14.0 ; 95 \%$ confidence interval, $1.2-164.7 ; P=.036)$. Other 


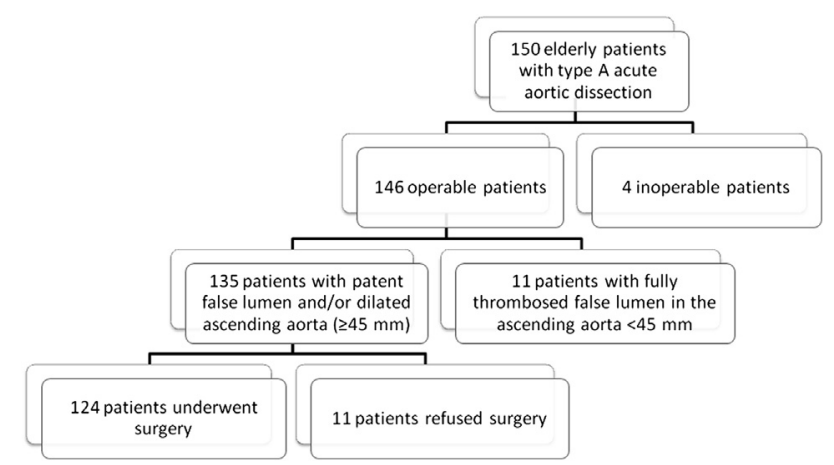

FIGURE 1. Decision making for our elderly patients with type A acute aortic dissection.

factors included in the model (age, female sex, cardiac tamponade, preoperative $\mathrm{CPR}$, preoperative neurologic deficit, myocardial ischemia, limb ischemia, previous cardiac surgery, preoperative hematocrit and serum creatinine levels, and TAR) were not significant predictors.

\section{Outcomes of Nonoperated Patients}

There were 26 patients ( $\geq 75$ years old) who were diagnosed with AAD during the same period who did not undergo surgery. All of 4 inoperable patients died on the day of admission. Of 11 patients with fully thrombosed false lumen and an ascending aorta $<45 \mathrm{~mm}, 1$ patient $(9.1 \%)$ died from pneumonia 59 days after admission. One-year actuarial survival rate of those patients was $90 \%$. Of 11 patients who refused surgery, 7 patients $(63.6 \%)$ died within 30 days after admission or before discharge. The causes of death were cardiac tamponade in 6

TABLE 1. Baseline characteristics of surgical patients and patients who refused surgery

\begin{tabular}{|c|c|c|c|}
\hline Variables & $\begin{array}{c}\text { Surgical } \\
\text { patients } \\
(\mathrm{n}=124)\end{array}$ & $\begin{array}{l}\text { Patients who } \\
\text { refused surgery } \\
(\mathbf{n}=\mathbf{1 1})\end{array}$ & $\begin{array}{c}P \\
\text { value }\end{array}$ \\
\hline Mean age \pm SD & $78.6 \pm 3.4$ & $83.9 \pm 6.1$ & $<.001$ \\
\hline Age $\geq 80$ y, n (\%) & $34(27.4)$ & $7(63.6)$ & .019 \\
\hline Women, n (\%) & $94(75.8)$ & $7(63.6)$ & .468 \\
\hline Cardiac tamponade, $\mathrm{n}(\%)$ & $31(25.0)$ & $2(18.2)$ & .732 \\
\hline $\begin{array}{l}\text { Required cardiopulmonary } \\
\text { resuscitation, } \mathrm{n}(\%)\end{array}$ & $8(6.5)$ & $0(0)$ & .626 \\
\hline Neurologic deficit, n (\%) & $15(12.1)$ & $0(0)$ & .611 \\
\hline Myocardial ischemia, n (\%) & $11(8.9)$ & $0(0)$ & .599 \\
\hline Limb ischemia, $\mathrm{n}(\%)$ & $4(3.3)$ & $1(9.1)$ & .351 \\
\hline Previous cardiac surgery, n (\%) & $3(2.4)$ & $1(9.1)$ & .291 \\
\hline $\begin{array}{l}\text { Mean preoperative } \\
\text { hematocrit, } \%, \pm \mathrm{SD}\end{array}$ & $33.7 \pm 5.8$ & $29.2 \pm 7.6$ & .016 \\
\hline $\begin{array}{l}\text { Mean preoperative creatinine, } \\
\mathrm{mg} / \mathrm{dL}, \pm \mathrm{SD}\end{array}$ & $0.9 \pm 0.6$ & $1.2 \pm 1.2$ & .192 \\
\hline $\begin{array}{l}\text { Fully thrombosed false } \\
\text { lumen, } n(\%)\end{array}$ & $39(31.4)$ & $4(36.4)$ & .743 \\
\hline
\end{tabular}

TABLE 2. Operative data and outcomes

\begin{tabular}{lc}
\hline \multicolumn{1}{c}{ Variables } & Total $(\mathbf{n}=\mathbf{1 2 4})$ \\
\hline Ascending aortic/hemiarch replacement, $\mathrm{n}(\%)$ & $106(85.5)$ \\
Total arch replacement, $\mathrm{n}(\%)$ & $18(14.5)$ \\
Concomitant procedures, $\mathrm{n}(\%)$ & $28(21.8)$ \\
Bentall operation & 9 \\
Aortic valve replacement & 4 \\
Coronary artery bypass grafting & 11 \\
Other procedures & 6 \\
Mean cardiopulmonary bypass time, min; $\pm \mathrm{SD}$ & $128.7 \pm 40.9$ \\
Mean aortic crossclamp time, min; $\pm \mathrm{SD}$ & $91.3 \pm 34.4$ \\
Operative death, n (\%) & $6(4.8)$ \\
Stroke, $\mathrm{n}(\%)$ & $22(17.7)$ \\
Respiratory failure, $\mathrm{n}(\%)$ & $27(21.8)$ \\
Reexploration for bleeding, $\mathrm{n}(\%)$ & $4(3.2)$ \\
Deep sternal infection, $\mathrm{n}(\%)$ & $4(3.2)$ \\
Median intensive care unit stay, d & 4 \\
Median length of hospital stay, d & 21 \\
Prolonged hospitalization $(>30 \mathrm{~d}), \mathrm{n}(\%)$ & $25(20.1)$ \\
\hline$S D$, Standard deviation. &
\end{tabular}

patients and respiratory failure in 1 patient. Two more patients died of cardiac tamponade during the follow-up period ( 2 months and 9 months after onset). One-year actuarial survival rate of those patients was $10.0 \%$.

\section{DISCUSSION}

In our elderly surgical cohort, the operative mortality was $4.8 \%$, and 1-, 3-, and 5-year actuarial survival rates were estimated at $89.3 \%, 83.6 \%$, and $79.1 \%$, respectively. Previous studies have reported operative mortalities in elderly

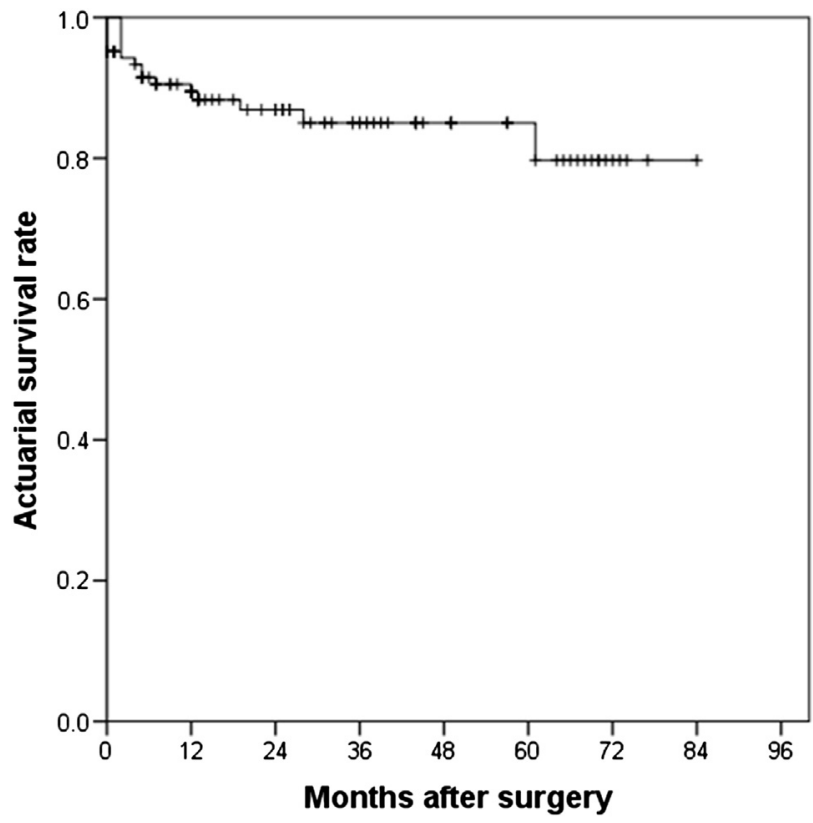

$\begin{array}{lllllllll}\text { No. at risk } & 124 & 86 & 58 & 37 & 23 & 15 & 4 & 1\end{array}$

FIGURE 2. Survival curve of surgical patients. 
patients (the definition of elderly patient varies in each study) with $\mathrm{AAD}$ ranging from $13.3 \%$ to $45.6 \%$ (some studies showed 30-day or in-hospital mortality instead of operative mortality). ${ }^{1,3-7}$ Five-year survival rates of surgical elderly patients with AAD have been reported previously, from $44 \%$ to $48.5 \%{ }^{3,4}$ Our results are superior to those reported previously, which might be because of our high surgical volume of AAD. Although the association of surgical volume with outcomes for AAD has not been shown, we experienced 422 emergency open surgeries for AAD during 6.5 years at a single center, and we have established an efficient process of diagnosis and treatment of AAD.

All of 6 operative deaths were related to stroke. The incidence of stroke was $17.7 \%$ (22/124). Advanced age is known as a risk factor of neurologic complications after surgical treatment of AAD. ${ }^{3,7}$ Our study has shown that preoperative CPR and previous cardiac surgery were significant predictors of stroke in elderly surgical patients with AAD. Preoperative hemodynamic compromise requiring CPR could lead to a significant decrease in cerebral perfusion. Quick diagnosis and decision making of surgery for AAD are critical to minimize a chance of preoperative hemodynamic compromise. However, it is often difficult to make a diagnosis of AAD because typical symptoms and signs of AAD, such as abrupt onset of back pain, murmur of aortic regurgitation, and pulse deficits, are less common in elderly patients compared with young patients. ${ }^{11}$ Previous cardiac surgery is known as a risk factor of surgery for AAD. ${ }^{12,13}$ However, emergency surgery has been shown to demonstrate better outcomes than conservative management even in those patients. ${ }^{12,13}$ More importantly, nearly half of our patients who had stroke retrieved their neurologic function and activities of daily living after rehabilitation. Considering this fact and our low operative mortality, emergency surgery can be the first choice for most elderly patients with AAD despite a high incidence of perioperative stroke.

In our elderly surgical cohort, TAR was performed in $14.5 \%$ of patients. During the same period, TAR was performed in $31.9 \%$ of younger patients ( $<75$ years old). Although our previous study has shown excellent outcomes of TAR, ${ }^{10}$ we do not use this extended operation excessively in emergency surgery of elderly patients. Hata and colleagues $^{14}$ reported excellent results of ascending aortic/hemiarch replacement with moderate hypothermia in octogenarians with AAD. Previous studies showed that a prolonged operation may increase mortality resulting from coagulopathy, cerebral ischemia, infection, and multiple organ failure. ${ }^{15,16}$ A simple, quick operation may be favorable for elderly patients with AAD.

In this study, a significant number of patients required prolonged hospitalization after surgery, and 6 patients $(4.8 \%)$ developed significantly impaired activities of daily living after surgery and became bedridden. Hata and colleagues ${ }^{5}$ described that $17 \%$ of their octogenarian $\mathrm{AAD}$ patients who underwent surgery became bedridden, and that their families were subjected to physical, mental, and financial stress. The family should be made aware of this possible undesirable outcome before surgery. Family support is another important factor to consider in the process of decision making for elderly patients, as well as patients' comorbidity and frailty. In addition, ethical issues make decision making difficult for elderly patients with AAD. ${ }^{17}$

The optimal therapeutic strategy for type A AAD with fully thrombosed false lumen (noncommunicating aortic dissection or intramural hematoma) is controversial. Some studies have recommended early surgery for all patients with type A intramural hematoma. ${ }^{18,19}$ On the other hand, other studies have shown favorable results of conservative treatment for type A intramural hematoma. ${ }^{20,21}$ Kaji and colleagues ${ }^{20}$ reported that the in-hospital mortality of medically treated type A intramural hematoma was $7 \%$ and that the 5-year survival rate was $90 \%$. Evangelista and colleagues $^{22}$ showed that a normal aortic diameter in the acute phase is the best predictor of intramural hematoma regression without complication. We applied conservative treatment when the ascending aortic diameter was $<45 \mathrm{~mm}$, and none of our patients developed cardiac or vascular events during the follow-up period. However, 1 patient died of pneumonia possibly related to prolonged bed rest for conservative management. Considering 0 operative mortality of our elderly patients with fully thrombosed false lumen, the optimal management is still controversial.

\section{Study Limitations}

There are limitations of an observational nature. This is a single-institute study and there is a patient selection bias. Because most of our AAD patients were referred from other hospitals, our cohort does not include some high-risk patients who were selected as a noncandidate for surgery at other hospitals. A larger study including surgical and nonsurgical AAD patients in the entire local area is necessary to obtain a complete view of AAD in the elderly.

\section{CONCLUSIONS}

Emergency open surgery for type A AAD in elderly patients resulted in low mortality but high incidences of stroke and prolonged hospitalization. Preoperative CPR and previous cardiac surgery were significant predictors of stroke. Emergency surgery is still the primary option for most elderly patients with type A AAD if the patient and family accept possible undesirable outcomes.

\section{References}

1. Mehta RH, Suzuki T, Hagan PG, Bassone E, Gilon D, Llovet A, et al. Predicting death in patients with acute type A aortic dissection. Circulation. 2002;105:200-6. 
2. David TE, Armstrong S, Ivanov J, Barnard S. Surgery for acute type A dissection. Ann Thorac Surg. 1999;67:1999-2001.

3. Hata M, Sezai A, Niino T, Yoda M, Unosawa S, Furukawa N, et al. Should emergency surgical intervention be performed for an octogenarian with type A acute aortic dissection? J Thorac Cardiovasc Surg. 2008;135:1042-6.

4. Piccardo A, Regesta T, Zannis K, Gariboldi V, Pansini S, Tapia M, et al. Outcomes after surgical treatment for type A acute aortic dissection in octogenarians: a multicenter study. Ann Thoracic Surg. 2009;88:491-7.

5. Santini F, Montalbano G, Messina A, D'Onofrio A, Casali G, Viscardi F, et al. Survival and quality of life after repair of acute type A aortic dissection in patients aged 75 years and older justify intervention. Eur J Cardiothorac Surg. 2006;29:386-91.

6. Trimarchi S, Eagle KA, Nienaber CA, Rampoldi V, Oh JK, Vincentiis CD, et al. Role of age in acute type A aortic dissection outcome: report from the International Registry of Acute Aortic Dissection (IRAD). J Thorac Cardiovasc Surg. 2010;140:784-9.

7. Rylski B, Suedkamp M, Beyersdorf F, Nitsch B, Hoffmann I, Blettner M, et al. Outcome after surgery for acute aortic dissection type A in patients over 70 years: data analysis from the German Registry for Acute Aortic Dissection Type A (GERAADA). Eur J Cardiothorac Surg. 2011;40:435-40.

8. Shimokawa T, Ozawa N, Takanashi S, Itoh T. Intermediate-term results of surgical treatment of acute intramural hematoma involving the ascending aorta. Ann Thorac Surg. 2008;85:982-6.

9. Matsushita A, Manabe S, Tabata M, Fukui T, Shimokawa T, Takanashi S. Efficacy and pitfalls of transapical cannulation for the repair of acute type A aortic dissection. Ann Thorac Surg. 2012;93:1905-9.

10. Matsuyama S, Tabata M, Shimokawa T, Matsushita A, Fukui T, Takanashi S. Outcomes of total arch replacement with stepwise distal anastomosis technique and modified perfusion strategy. J Thorac Cardiovasc Surg. 2012;143:1377-81.

11. Mehta RH, O'Gara PT, Bossone E, Nienaber CA, Myrmel T, Cooper JV, et al Acute type A aortic dissection in the elderly: clinical characteristics, management, and outcomes in the current era. J Am Coll Cardiol. 2002;40:685-92.
12. Klodell CT, Karimi A, Beaver TM, Hess PJ, Martin TD. Outcomes for acute type A aortic dissection: effects of previous cardiac surgery. Ann Thorac Surg. 2012; 93:1206-12

13. Estrera AL, Miller CC, Kaneko T, Lee TY, Walkes JC, Kaiser LR, et al. Outcomes of acute type A aortic dissection after previous cardiac surgery. Ann Thorac Surg. 2010;89:1467-74.

14. Hata M, Suzuki M, Sezai A, Niino T, Unosawa S, Furukawa N, et al. Less invasive quick replacement for octogenarians with type A acute aortic dissection. J Thorac Cardiovasc Surg. 2008;136:489-93.

15. Czerny M, Fleck T, Zimpfer D, Dworschak M, Hofmann W, Hutschala D, et al. Risk factors of mortality and permanent neurologic injury in patients undergoing ascending aortic and arch repair. J Thorac Cardiovasc Surg. 2003;126:1296-301.

16. Salis S, Mazzanti VV, Merli G, Salvi L, Tedesco CC, Veglia F, et al. Cardiopulmonary bypass duration is an independent predictor of morbidity and mortality after cardiac surgery. J Cardiothorac Vasc Anesth. 2008;22:814-22.

17. McKneally MF. "We didn't expect dementia and diapers": reflection on the Nihon experience with type A aortic dissection in octogenarians. J Thorac Cardiovasc Surg. 2008;135:984-5.

18. Tittle SL, Lynch RJ, Cole PE, Singh HS, Rizzo JA, Kopf GS, et al. Midterm follow-up of penetrating ulcer and intramural hematoma of the aorta. J Thorac Cardiovasc Surg. 2002;123:1051-9.

19. Uchida K, Imoto K, Takahashi M, Suzuki S, Isoda S, Sugiyama M, et al. Pathologic characteristics and surgical indications of superacute type A intramural hematoma. Ann Thorac Surg. 2005;79:1518-21.

20. Kaji S, Akasaka T, Horibata Y, Nishigami K, Shono H, Katayama M, et al. Longterm prognosis of patients with type A aortic intramural hematoma. Circulation. 2002;106:1248-52.

21. Song JK, Kim HS, Song JM, Kang DH, Ha JW, Rim SJ, et al. Outcomes of medically treated patients with aortic intramural hematoma. Am J Med. 2002;113:181-7.

22. Evangelista A, Mukherjee D, Mehta RH, O'Gara PT, Fattori R, Cooper JV, et al. Acute intramural hematoma of the aorta: a mystery in evolution. Circulation. 2005;111:1063-70. 